\title{
La poète en transit : étude du déplacement dans trois recueils de poésie franco-canadiens
}

\section{Véronique Arseneau}

Dans le contexte de la littérature franco-canadienne, c'est-à-dire de «l'ensemble des littératures de l'Acadie, de l'Ontario français et de l'Ouest francophone tout en excluant celle du Québec » (Cormier et Brun del Re, 2016, p. 53-54), les écrivaines sont doublement marginalisées par leur statut de femme et leur appartenance à une communauté linguistique minoritaire. Ce statut les enferme dans un espace doublement exigu ${ }^{1}$, dont elles tentent de s'affranchir par une écriture intimement liée au déplacement. Dans La poétique de l'espace, Bachelard (1972 [1957], p. 183) explique d'ailleurs que le soi se construit en réaction à ce qui l'entoure, et vice-versa : «[s]ans cesse les deux espaces, l'espace intime et l'espace extérieur viennent, si l'on ose dire, s'encourager dans leur croissance ». C'est donc souvent par le voyage que l'on peut se renouveler et se découvrir, même si celui-ci était, pendant un certain temps, inaccessible aux femmes. Selon Morency, den Toonder et Lintvelt (2006), cette situation s'explique par le fait que «le voyage a longtemps été l'apanage des hommes » et que «la recherche identitaire était également le privilège du sexe masculin ». Ils notent cependant que «[1]a littérature contemporaine montre que la femme fait de plus en plus la conquête de l'espace » (Morency et al., p. 6). Cette réalité est vrai non seulement pour les personnages féminins, mais aussi pour les auteures qui s'approprient leur place au sein de l'institution littéraire.

Cette conquête de l'espace par les femmes est certes présente dans des romans québécois et franco-canadiens, tels que les romans de la route ${ }^{2}$ ou, encore, les romans de voyage, mais

\footnotetext{
${ }^{1}$ Les termes «exigu» et «exiguïté » renvoient au concept développé par François Paré (1992) dans son incontournable essai Les littératures de l'exiguïté.

${ }^{2}$ Les road novels ou, en français, romans de la route, sont des romans où le voyage, la route, l'errance et la découverte de soi et de l'autre sont des thématiques omniprésentes. Le road novel le plus connu est sans aucun doute On the road (Sur la route, en français) du Franco-Américain Jack Kerouac (1973 [1957]).
} 
elle est également présente dans la poésie. En Ontario français et en Acadie, par exemple, c'est par une poésie décrivant le territoire que la littérature a vu le jour. Néanmoins, cette poésie est presque exclusivement écrite par des hommes ${ }^{3}$. Depuis, plusieurs poètes franco-canadiennes ont publié des recueils où l'espace et, surtout, le déplacement sont omniprésents.

Dans le cadre de notre thèse de maîtrise en lettres françaises (Arseneau, 2017), nous nous sommes intéressée au rôle du déplacement et du voyage dans les textes poétiques d'écrivaines franco-canadiennes. Nous avons analysé la représentation de l'espace, plus précisément celle du déplacement. Il s'agissait de cerner, dans ce contexte de double minorisation, le rôle joué par le déplacement dans la construction du soi féminin dans trois moments ciblés du parcours des personnages : l'ici, le transit et l'ailleurs. Pour ce faire, nous avons étudié la notion du déplacement dans des textes poétiques de trois écrivaines franco-canadiennes originaires du Nouveau-Brunswick, de l'Ontario et du Manitoba. Notre thèse avait pour objectif principal de voir de quelle façon la poésie des femmes en contexte francophone minoritaire aborde la construction de soi à l'aide d'une écriture poétique qui explore à la fois le déplacement ontologique et physique. Cet article présente donc un aperçu condensé de nos analyses faites à la maîtrise, notamment sur le rôle du déplacement et du voyage dans les trois recueils que nous avons étudiés : d'abord La Voyageuse, d'Andrée Lacelle; ensuite, Rues étrangères, de Sarah Marylou Brideau; enfin, Poste restante : cartes poétiques du Sénégal, de Lise Gaboury-Diallo. Il s'agira de cerner, dans ce contexte de double minorisation, le rôle joué par l'espace dans la construction du soi féminin des trois poètes en plein transit. Notre hypothèse est que les poèmes à l'étude décrivent la façon dont le passage d'un imaginaire du proche, de l'ici, à un imaginaire de l'autre, de l'ailleurs, mène à une redéfinition du soi féminin. Cette étude s'attardera particulièrement à l'espace transitoire, c'est-à-dire à ce moment entre l'ici et l'ailleurs.

\section{L'espace transitoire : une poésie de l'entre-deux}

Héritage des road novels et de la fascination de la poésie du paysage et du territoire, de l'appel de la route, du désir de changement et de la volonté d'améliorer sa condition, le

\footnotetext{
${ }^{3}$ Nous pensons, entre autres, à Patrice Desbiens et à Jean Marc Dalpé pour l'Ontario ainsi qu'à Guy Arsenault et à Herménégilde Chiasson pour l'Acadie.
} 
déplacement est un concept omniprésent en littérature franco-canadienne. Le déplacement, sous sa forme la plus simple, représente une migration d'un sujet d'un point $\mathrm{A}$ à un point B. Dans leur poésie, Andrée Lacelle, Lise Gaboury-Diallo et Sarah Marylou Brideau se servent du déplacement non pas uniquement comme un déplacement géographique des personnages, mais surtout comme un déplacement ontologique, c'est-à-dire interne. Dans les trois recueils étudiés, nous assistons à une progression interne des personnages dans un espace dit «transitoire». Ainsi, nous appelons «transit» ce moment dans le texte où la poète bascule dans l'entre-deux, dans l'incertain, voire souvent dans un déplacement qui a pour but final un ailleurs plus ou moins défini. Ce changement dans les poèmes, difficile à cerner, permet au lecteur de mieux saisir l'importance du déplacement dans les poèmes, de la construction d'un passage ontologique vers un ailleurs, vers autre chose, sans toutefois y voir une certaine finalité. Car le transit et le déplacement sont continus, non fixes, et créent souvent un décalage. En fait, comme l'explique Sibony (1991, p. 228),

se déplacer, supporter le passage entre-deux, c'est pouvoir accomplir en soi le geste de passer; le consentement au passé ; l'acte crucial de faire le pas. [...] Il faut de l'espace intérieur pour faire le pas, pour se dépasser un peu, pour se déplacer, au risque d'être déplacé; décalage dans sa propre identité.

Voyons maintenant, à l'aide d'analyses de poèmes ciblés, de quelle façon cet espace transitoire est construit poétiquement et de quelle façon, dans une certaine mesure, cet espace de l'entre-deux permet aux personnages-poètes de s'affranchir et de devenir agentes ${ }^{4}$, c'est-à-dire de «se construire une identité cohérente, de s'autodéterminer et d'agir avec discernement et en accord avec ses valeurs et ses désirs » (Cardinal, 2000, p. 30).

\section{Sarah Marylou Brideau}

Dans son deuxième recueil, intitulé Rues étrangères, la poète acadienne Sarah Marylou Brideau (2004) décrit un personnage qui chemine entre deux espaces réels : Moncton et Fredericton. Ces deux villes, dans l'imaginaire collectif acadien, renvoient à deux

\footnotetext{
${ }^{4}$ Les théories de l'agentivité (agency), d'abord développées par Gardiner (1995) en littérature, résident « dans la capacité d'agir dans sa vie, de réaliser son potentiel malgré les difficultés sociales, familiales et culturelles » (Havercroft, 1999, p. 94). Il s'agit ainsi de «la capacité que possède un individu de faire des changements dans sa conscience individuelle, dans sa vie personnelle et dans la société » (Cardinal, 2000, p. 30).
} 
communautés distinctes : francophones et anglophones. Au moment de publier ce recueil, Brideau est partie de Moncton pour aller étudier à Fredericton « où elle étudie le Français à St. Thomas University » (2004, quatrième de couverture). Ainsi, dans ce déplacement géographique bien réel, on observe aussi une transition interne chez le personnage, celle-ci changeant de dénomination, passant d' «étrangère » à « locale ». En s'appropriant l'espace étranger, dans ce cas-ci, celui de Fredericton, la poète dans le recueil reconstruit l'espace, qui devient sien.

L'espace transitoire chez Brideau (2004) est particulièrement présent dans le poème «Transitions ». Ce poème est le premier du recueil où Brideau décrit les aspects plus négatifs découlant du passage, qu'il soit géographique ou ontologique. Le poème s'ouvre sur la strophe suivante, montrant que la transition est difficile pour la poète :

Traverser les étapes

tumultueuses

une tempête

the damage has been done (p. 14)

Les vers suivants : «Changer de direction / et tout abandonner / me semble impossible » (Brideau, 2004, p. 14), énoncent un certain doute face à la voie choisie, presque comme une forme de regret. En fait, la poète semble tiraillée entre le désir d'abandonner et le besoin de «[c]ontinuer / à se relever» (Brideau). Enfin, même si celle-ci se sent lancée dans le vide et arrachée au confort de son chez-soi lorsqu'elle dit: "free falling / déracinée » (Brideau), il n'empêche qu'elle sent que ce changement est nécessaire : « [f]aire place à la transition / ouvrir la porte aux choses nouvelles / accueillir une difficile transition » (Brideau). Le poème joue ainsi avec cette tension interne de la poète.

Ainsi, dans ce poème, la notion de transit se trouve très intériorisée, et ce transit intérieur se retrouve également tout au long du recueil. Avec «Transitions », on constate que la poète semble accepter avec difficulté le changement, mais comprend que celui-ci est nécessaire. Le champ sémantique employé par Brideau (2004) renvoie d'ailleurs au déplacement et à l'espace, au territoire, ne serait-ce qu'avec les termes suivants: « Traverser », « tempête », «sky», «land», « direction », «transition », « déracinée » (Brideau). 
Cette inquiétude et cette peur du changement se poursuivent dans le poème suivant, intitulé «J'ai fermé les yeux », où la poète affirme avoir «résisté au changement» et « fermé les yeux », ne voulant pas « passer à autre chose» (Brideau, 2004, p. 15). Encore une fois, le début du poème est assez fermé. Ce n'est qu'après une certaine introspection que la poète s'ouvre au monde des possibles : «Les yeux fermés / la tête pourrie / mon cœur s'ouvrit»(Brideau). Enfin, après cette ouverture, la poète peut enfin partir à nouveau :
Alors je repris la route
vers le chemin qui s'éclairci [sic]
sur un horizon guidé
par la quiétude de la prospérité (Brideau)

L'espace transitoire chez Brideau (2004) sert donc de pont entre le besoin de stabilité, mais aussi celui de changement constant chez le personnage, qui cherche toujours à la fois un chez-soi et une nouvelle aventure.

\section{Andrée Lacelle}

Andrée Lacelle, poète franco-ontarienne de notre corpus, est celle qui écrit une poésie des plus «hermétiques » (Tessier, 2001, p. 92), une poésie de l'intime. Ne faisant aucune référence à aucun lieu géographique réel, le rapport au déplacement dans La Voyageuse est plus métaphorique que celui des deux autres recueils étudiés. C'est d'ailleurs par la métaphore et l'analogie que le personnage éponyme — «la voyageuse » du recueil — réussit à s'affranchir.

Chez Lacelle, l'espace transitoire se retrouve dans la série de poèmes « Le temps profond ». Cette série, qui comprend huit poèmes, débute en inscrivant la voyageuse dans l'entre-deux (Sibony, 1991) :

Elle avance dans l'entre-deux

se recollent les séquences de l'époque de veille quand loin des routes immédiates

dans l'instant du regard toutes choses s'exposent (Lacelle, 1995, p. 29, nous soulignons). 
Ici, cet espace mitoyen, à l'écart de tout, permet à la voyageuse de mieux (se) comprendre. Ce court poème mentionne aussi un espace « loin des routes immédiates », ce qui renvoie à un certain isolement, mais de façon positive, un recueillement loin de l'influence des autres, permettant à la voyageuse de se ressourcer.

Dans la suite poétique, nous remarquons aussi la présence du temps, et non de l'espace, ce qui est intéressant, et ce, parce que le transit n'est pas nécessairement un lieu, mais plutôt un moment, une période, voire un cheminement. Dès le deuxième poème de cette série, c'est un temps « rompu », « en exil » et « inhabité » qui fait son apparition (Lacelle, 1995, p. 30). Plus loin, «le temps décline / perpétuel décalage entre le monde et soi » (Lacelle, p. 31). Ici, Lacelle semble entretenir un rapport particulier au temps comme si celui-ci lui échappait constamment.

Dans La Voyageuse, la série de poèmes «Le temps profond » tend à montrer la façon dont le temps et l'espace se rejoignent dans les yeux de la voyageuse. Les deux thèmes se côtoient, voire se confondent, au niveau lexical : «le temps pulvérise l'espace d'hier» (Lacelle, 1995, p. 34, nous soulignons). Ainsi comme le souligne Voldeng (1995),

nous avons [dans ce recueil ] un voyage dans le temps, la géographie, le voyage mental d'une femme qui avance dans «l'entre-deux » [...], d'une nomade dans un désert brûlant, d'une "pérégrine» hantée par le soleil des mystiques. [...] [L]a voyageuse écrit et ses poèmes sont les marches vers le lieu de la communion mystique où se résorbent temps et espace (p. 42)

Enfin, le poème qui clôt cette section de La Voyageuse est évocateur lorsque la poète affirme que «ce lieu n'est pas un lieu » (Lacelle, 1995, p. 36). Ce lieu qui n'en est pas un devient en quelque sorte l'instant des révélations, de la compréhension. Il s'agit plutôt d'un moment de recueillement pour la voyageuse dont la durée ne peut être définie, tout comme l'espace. D'ailleurs, «l'œuvre d'Andrée Lacelle nous invite [...] à pénétrer un univers sans apothéose, béni par l'inachèvement » (Paré, 2015, p. 15). Cette idée d'inachèvement, de continuité dans l'œuvre de Lacelle, se traduit par une quête infinie vers le Vrai, vers le Sens, et non vers une quête purement identitaire comme cela est habituellement le cas en poésie franco-canadienne. La voyageuse du recueil de Lacelle est donc en constante quête, en constant déplacement. 


\section{Lise Gaboury-Diallo}

Lise Gaboury-Diallo, auteure franco-manitobaine, publie en 2005 Poste restante: cartes poétiques du Sénégal, un court recueil qui relate son voyage avec sa famille au Sénégal, lieu de naissance de son mari. Son rapport à l'espace est ainsi tout à fait différent : en sachant qu'il y a un retour envisagé, son expérience du déplacement est à l'opposé de ce que décrivent Lacelle et Brideau. La poésie de Gaboury-Diallo est ainsi, comme le souligne Paré (2011, p. 12), « une exploration lumineuse du départ, de la distance et surtout de la rencontre. »

Gaboury-Diallo aborde l'espace transitoire dans Poste restante par la forme même du recueil. L'analyse de Hotte (2010) décrit Poste restante comme un recueil de poèmes se situ[a]nt à la confluence de trois genres : la carte postale, le journal intime et la poésie. Ce genre hybride, qu'elle nomme «cartes poétiques », de même que l'appareil paratextuel découle de la nécessité d'innover formellement face à une réalité autre et épouse ainsi les transformations identitaires que le voyage entraîne et qui sont explorées dans les poèmes. Ceux-ci touchent à quatre éléments : le déplacement, la nouvelle réalité, la différence et la transformation. (p. 107)

Ces quatre éléments identifiés par Hotte pourraient correspondre aux quatre « moments » clés du recueil de Gaboury-Diallo (2005) qui permettent à la poète de passer de l'ici à l'ailleurs. Il y a, évidemment, au début du recueil un réel déplacement géographique, du Manitoba au Sénégal, puis une acclimatation à une nouvelle réalité, une constatation de la différence dans le rapport entre le soi et l'autre et, enfin, une transformation intérieure en réaction aux expériences vécues. Cette transformation se produit en filigrane dans le texte. Dans plusieurs poèmes, la poète devient simple observatrice, un peu en retrait, et absorbe les nombreuses expériences qu'elle vit lors de son voyage. Ainsi, «[p]eu lui importe en effet de tout voir ou de tout comprendre, c'est une expérience sensuelle et profondément ressentie qui occupe la poète visant à établir des liens émotionnels ou affectifs avec le pays et ses habitants »(Lohka, 2012, p. 164).

Il n'y a pas que l'espace qui prédomine dans l'écriture de Gaboury-Diallo (2005). Selon Paré (2011, p. 12), la poète « chercherait souvent à évoquer de manière allusive [d]es scènes d'étrangeté et d'accueil ». Dans le poème, «je commencerai après tout », la poète s'arrête, pendant le voyage, pour réfléchir à son processus d'écriture. Ce poème 
autoréflexif entame une analyse sur le recueil en devenir de Gaboury-Diallo, ce qui explique sa longueur par rapport aux autres poèmes, qui contiennent plus de descriptions des lieux visités que d'introspection. Ainsi, alors qu'il est en plein transit, le «je» énonciateur de ce poème réfléchit à la fois au présent et à l'avenir. La poète envisage d'ailleurs déjà qu'elle écrira différemment, « une écriture autre » (Gaboury-Diallo, 2005 p. 49) à la fin du voyage, reconnaissant ici l'influence des autres et des expériences qu'elle a vécues lors des derniers jours passés au Sénégal.

C'est également grâce à ce changement au sein de son écriture qu'elle remerciera, non seulement dans ce poème, mais aussi dans d'autres qui suivront, l'influence positive de ceux qui ont croisé sa route, d'où la «mappemonde de vœux » (Gaboury-Diallo, 2005, p. 49) ou encore le début du poème « et dire merci » : « et dire merci / c'est trop peu / pour ceux qui voyagent » (Gaboury-Diallo, p. 56), qui est accompagné d'une dédicace à «tous ceux qui nous ont accueillis hébergés nourris soignés dorlotés un merci qui ne suffira jamais à tout dire» (Gaboury-Diallo). Il semble donc que ce changement interne, transitoire, de la poète dans Poste restante, est directement lié aux rencontres avec autrui.

\section{Conclusion}

En somme, la poésie de Brideau (2004) vogue entre deux endroits bien réels, mais ces lieux sont peu importants, puisque la poète accorde une grande partie de son recueil au transit, au cheminement interne qui se produit lors de nombreux déplacements. Pour Gaboury-Diallo (2005), c'est par le voyage que se produit la construction du transit. Enfin, alors que la poésie de Brideau et de Gaboury-Diallo est ancrée dans des lieux bien réels, celle de Lacelle (1995) se distingue par son utilisation du déplacement comme métaphore tout au long du recueil. Ainsi, les trois poètes étudiées utilisent toutes l'espace, qu'il soit réel ou fictif, géographique ou ontologique, afin d'illustrer un cheminement intérieur, une certaine construction, voire une reconstruction de soi par le biais de leur écriture. Malgré leurs différences et leur parcours distinct, ces trois auteures de la francophonie canadienne semblent toutes accorder une importance particulière à une écriture du transit dans leur poésie, écriture qui permet d'atteindre l'Autre, l'ailleurs, mais surtout soi-même. À la fois tournée vers le lointain et recentrée sur l'intérieur / le soi, la poésie du transit, de l'entre-deux permet de mieux comprendre le rapport interne/externe ainsi que les liens et 
les thématiques qui unissent les différentes littératures francophones minoritaires au Canada au féminin.

L'auteure tient à préciser que cette recherche a été financée par le Conseil de recherches en sciences humaines du Canada (CRSH) en 2016-2017 dans le cadre de sa thèse de maîtrise (Arseneau, 2017).

\section{Références}

Arseneau, V. (2017). Le déplacement au féminin : la poésie franco-canadienne en quête d'un soi et d'un ailleurs. Thèse de maîtrise. Université d'Ottawa, Ontario.

Bachelard, G. (1972 [1957]). La poétique de l'espace. Paris, France : Presses universitaires de France.

Brideau, Sarah Marylou (2004). Rues étrangères. Moncton, Nouveau-Brunswick: Éditions Perce-Neige.

Cormier, P. et Brun del Re, A. (2016). Vers une littérature franco-canadienne? Bases conceptuelles et institutionnelles d'un nouvel espace littéraire. Dans Thibeault, J., Long, D., Nyela, D. et Wilson, J. (dir.), Au-delà de l'exiguïté. Échos et convergences dans les littératures minoritaires. Moncton, Nouveau-Brunswick : Éditions Perce-Neige, 53-75.

Cardinal, J. (2000). Suzanne Jacob et la résistance aux «fictions dominantes » : figures féminines et procédés rhétoriques rebelles. Mémoire de maîtrise. Université du Québec à Montréal, Québec.

Gaboury-Diallo, L. (2005). Poste restante : cartes poétiques du Sénégal. Saint-Boniface, Manitoba : Éditions du Blé.

Gardiner, J. (dir.) (1995). Provoking Agents: Gender and Agency in Theory and Practice. Urbana, Illinois : University of Illinois Press.

Havercroft, B. (1999). Quand écrire, c'est agir : stratégies narratives d'agentivité féministe dans Journal pour mémoire de France Théoret. Dalhousie French Studies 47, «Écriture de soi au féminin », 93-113.

Hotte, L. (2010). Le goût de l'ailleurs dans la poésie de Lise Gaboury-Diallo, Études Canadiennes (69), 107-122.

Kerouac, J. (1973 [1957]). Sur la route. Paris, France : Flammarion. 
Lacelle, A. (1995). La Voyageuse. Sudbury, Ontario : Éditions Prise de parole.

Lohka, E. (2012). Une poésie d'/de l'ailleurs en Amérique du Nord : Lise Gaboury-Diallo.

Dans Young, L. (dir.), Langages poétiques et poésie francophone en Amérique du Nord, Québec, Québec : Presses de l’Université Laval, 161-169.

Morency, J., den Toonder, J. et Lintvelt, J. (dir.) (2006). Romans de la route et voyages identitaires. Québec, Québec : Éditions Nota bene.

Paré, F. (1992). Les littératures de l'exiguïté. Hearst, Ontario : Éditions du Nordir.

Paré, F. (2011). Lise Gaboury-Diallo : les figures inépuisables du lointain. Nuit blanche (124), 12-14.

Paré, F. (2015). Préface. Dans Lacelle, A. Sol Ciel Ciels Sols (p. 7-15). Sudbury, Ontario : Éditions Prise de parole.

Sibony, D. (1991). Entre-deux. L'origine en partage. Paris, France : Éditions du Seuil.

Tessier, J. (2001). Andrée Lacelle et la critique, Francophonies d'Amérique (11), 91-101.

Voldeng, É. (1995). Andrée Lacelle : une «pérégrine» en quête d'Absolu. Liaison (83), 42. 